\title{
A Patient Developing Chemotherapy-Induced Peripheral Neuropathy in Which Development Correlated with Changes in Current Perception Threshold and Plasma Neurotrophins and Substance P Levels
}

\author{
Maiko MACHIDA $^{* 1}$, Shinya TAMAKI ${ }^{* 2}$, Yuta FUKAI ${ }^{* 2}$, Hiroyuki KAWAGUCHI ${ }^{* 2}$, \\ Masayuki ENDO $^{* 2}$, Yasuo TAKAHASHI ${ }^{* 3}$ and Nobuo INOTSUME ${ }^{* 4}$
}

\section{Introduction}

Chemotherapy-induced peripheral neuropathy (CIPN) is a significant side effect and may cause patients to discontinue chemotherapy, even those who have a good response. Although combination therapy of fluorouracil (5-FU) and folinic acid (LV) with oxaliplatin(FOLFOX) has been established as a standard first-line therapy for advanced colorectal cancer, one of the limiting toxicities is sensory neuropathy due to oxaliplatin ${ }^{1)}$. The development of CIPN is a potential treatment-limiting consideration $^{2)}$, because adequate supportive therapy for CIPN is not yet available. The Stop-and-Go strategy, in which the therapy is stopped before severe neurotoxicity develops after six cycles followed by another regimen without oxaliplatin and later reintroduction of the former regimen, reduces the risk of grade 3 to 4 toxicity $^{3)}$. However, grading of the toxicity can be difficult to judge, considering the limitation of one's activities of daily living depends on individual ambiguous complaints without obvious quantitative signs and symptoms, especially in the early stage of severity. Thus, we focused on evaluation of the severity of CIPN in the early stage to predict future severity and prepare for decisions to change the regimen.

Simozuma et al. reported a tendency to underreport and underestimate the severity of peripheral neuropathy in grading with the National Cancer Institute Common Toxicity Criteria $(\text { NCI-CTC })^{4)}$. Even if various patient-based grading scores of the subscale for CIPN are also available ${ }^{5-7)}$, the grading score for clinical responsiveness may still be difficult to determine. As for rigorous diagnosis, invasive electrophysiological nerve conduction studies (NCS) are time-consuming. Non-invasive measurement of the current perception threshold (CPT) in peripheral regions is more feasible and useful for the detection, screening and management of diseases of peripheral neuropathy ${ }^{8)}$. Unlike NCS, selective quantitative evaluation of specific nerve types is possible by stimulating CPT at different frequencies to differentiate mono-neuropathies from poly-neuropathies ${ }^{9)}$. A trade-off is made between sensitivity and specificity on CPT values. Thus, available biomarkers are required for compensate for these problems. Our aim was to measure whether circulating levels of some available biomarkers in peripheral blood, when accompanied by any changes in CPT values, correspond to the initial clinical responsiveness of CIPN in the early stage.

Nerve growth factor (NGF) and brain-derived neurotropic factor (BDNF), as members of the neuro-protective neurotrophin family, may contribute to the pathobiology of neuropathic pain ${ }^{10)}$. In sensory neurons, BDNF and substance $\mathrm{P}(\mathrm{SP})$, as neurotransmitters involved in pain sensitivity ${ }^{11)}$, depend on the availability of $\mathrm{NGF}^{10,12)}$. Meanwhile, the normal neuronal suppressive effects would shift to an opposite effect of hyperexcitability in allodynia, making CIPN complicated. BDNF and interferon (IFN)- $\gamma$-related allodynia in conditions of sustained neuropathy have been considered ${ }^{13)}$. Simultaneous evaluation of these measureable peptides in peripheral blood as possible candidate biomarkers, with monitoring of CPT values and grading scores of clinical responsiveness, thus provide beneficial information about CIPN in the early stage.

\section{Case Presentation}

A 64-year-old woman who was suffering from upper colon cancer and multiple hepatic metastases (stage IV) was enrolled in this study. The patient was treated every 2 weeks with two cycles of chemotherapy consisting of FOLFOX $+\mathrm{B}$ (oxaliplatin $85 \mathrm{mg} / \mathrm{m}^{2}, \quad$ LV $200 \mathrm{mg} / \mathrm{m}^{2}$, and bevacizumab $130 \mathrm{mg}$ 2-h infusion, 5 -FU $400 \mathrm{mg} / \mathrm{m}^{2}$ bolus infusion plus $2,400 \mathrm{mg} / \mathrm{m}^{2}$ 46-h continuous infusion). The patient was discharged 1 week after the second cycle of chemotherapy and moved to a local hospital, leading to termination from this study. Doses in the first and second cycles were $100 \%$ and $90 \%$ of standard doses, respectively. Clinical examination data were monitored during the course until 1 week after second cycle (Table). The patient was almost afebrile, and undernutrition was ruled out based on albumin and total protein levels.

Key words : chemotherapy-induced peripheral neuropathy, current perception thresholds, nerve growth factor, brain-derived neurotropic factor, substance $\mathrm{P}$, interferon- $\gamma$

${ }^{* 1}$ Department of Pharmacotherapy, Hokkaido Pharmaceutical University School of Pharmacy, Japan ${ }^{* 2}$ Department of Pharmacy, National Hospital Organization Hokkaido Cancer Center, Japan $\quad{ }^{* 3}$ Department of Gastroenterology, National Hospital Organization Hokkaido Cancer Center, Japan $\quad{ }^{* 4}$ Department of Clinical Pharmacology, Hokkaido Pharmaceutical University School of Pharmacy, Japan Address for correspondence : MACHIDA M. Department of Pharmacotherapy, Hokkaido Pharmaceutical University School of Pharmacy, 7-154-1 Maeda, Teineku, Sapporo 006-8590, Japan E-mail : machida-m@hokuyakudai.ac.jp

Manuscript received September 16, 2015 ; revised December 21, 2015 and January 22, 2016 ; accepted January 23, 2016

ISSN 0388-1601 
Table Clinical examination data

\begin{tabular}{lcccccc}
\hline & Baseline & $\begin{array}{c}1 \text { day } \\
\text { after } \\
1^{\text {st }} \text { cycle }\end{array}$ & $\begin{array}{c}1 \text { week } \\
\text { after } \\
1^{\text {st }} \text { cycle }\end{array}$ & $\begin{array}{c}1 \text { day } \\
\text { after } \\
2^{\text {nd }} \text { cycle }\end{array}$ & $\begin{array}{c}5 \text { day } \\
\text { after } \\
2^{\text {nd }} \text { cycle }\end{array}$ & $\begin{array}{c}1 \text { week } \\
\text { after } \\
2^{\text {nd }} \text { cycle }\end{array}$ \\
\hline Albumin $(\mathrm{g} / \mathrm{dL})$ & 4 & 4.3 & 3.7 & 3.9 & 4.2 & - \\
$\mathrm{AST}(\mathrm{IU} / \mathrm{L})$ & 34 & 35 & 29 & 26 & 41 & - \\
$\mathrm{ALT}(\mathrm{IU} / \mathrm{L})$ & 11 & 12 & 12 & 10 & 18 & - \\
$\mathrm{T} . \mathrm{Bilimubin}(\mathrm{mg} / \mathrm{dL})$ & 0.66 & 1.3 & 0.44 & 0.52 & 1.1 & - \\
$\mathrm{BUN}(\mathrm{mg} / \mathrm{dL})$ & 10.3 & - & 9.8 & - & 13.5 & - \\
$\mathrm{Scr}(\mathrm{mg} / \mathrm{dL})$ & 0.52 & 0.51 & 0.46 & 0.49 & 0.53 & - \\
$\mathrm{WBC}\left(10^{3} / \mu \mathrm{L}\right)$ & 2.74 & 2.89 & 2.67 & 2.74 & 1.63 & 8.03 \\
$\mathrm{RBC}\left(10^{4} / \mu \mathrm{L}\right)$ & 370 & 412 & 351 & 364 & 414 & 347 \\
$\mathrm{Hb}(\mathrm{g} / \mathrm{dL})$ & 11.8 & 13.2 & 11.4 & 11.5 & 13.1 & 11.3 \\
$\mathrm{Plt}\left(10^{4} / \mu \mathrm{L}\right)$ & 20.9 & 21.2 & 17.1 & 19.1 & 18.8 & 15.7 \\
$\mathrm{TP}(\mathrm{g} / \mathrm{dL})$ & 6.4 & 7.2 & 6.0 & 6.3 & 6.9 & - \\
$\mathrm{BT}\left({ }^{\circ} \mathrm{C}\right)$ & 36.5 & 37.0 & 36.8 & 36.6 & 37 & 36.7 \\
$\mathrm{CEA}(\mathrm{ng} / \mathrm{mL})$ & 13 & 15.6 & - & - & - & 14.7 \\
$\mathrm{CA} 19-9(\mathrm{U} / \mathrm{mL})$ & 205 & 296 & - & - & - & 234 \\
\hline
\end{tabular}

AST, Aspartate transaminase ; ALT, alanine transaminase ; BUN, blood urea nitrogen ; Scr, serum creatinine ; WBC, white blood cell ; RBC, red blood cell ; Hb, hemoglobin; Plt, platelet ; TP, total protein ; BT, body temperature ; CEA, carcinoembryonic antigen ; CA19-9, carbohydrate antigen 19-9.
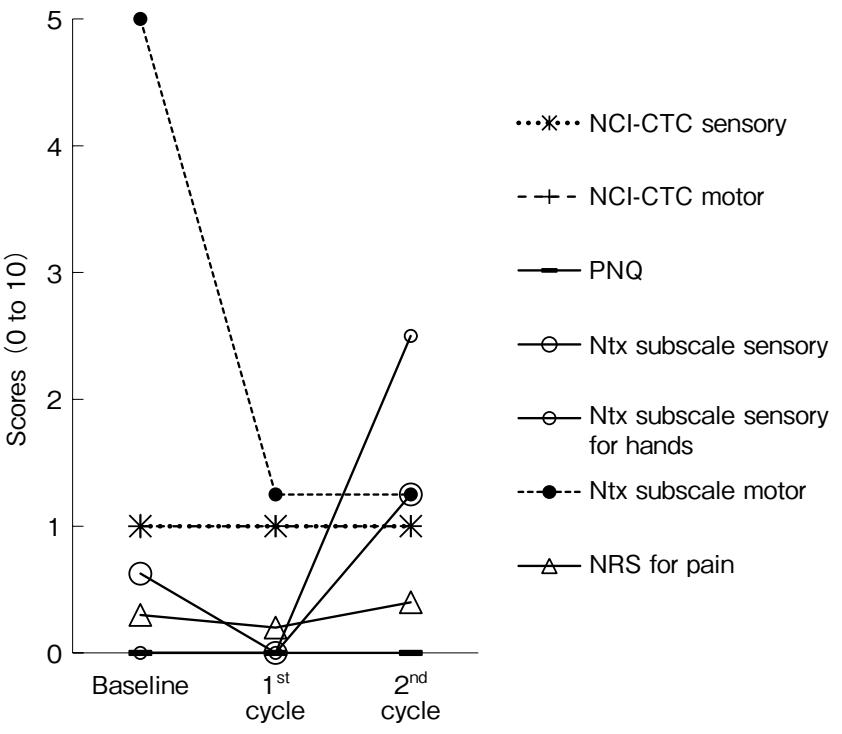

Figure 1 Grading scores for clinical responsiveness

NCI-CTC, National Cancer Institute Common Toxicity Criteria ; PNQ, patient neurotoxicity questionnaire; $\mathrm{Ntx}$, neurotoxicity from functional assessment of cancer therapy/gynecologic oncology group-neurotoxicity ; NRS, numerical rating scale.

After a four-week observation period, the patient underwent CPT examinations and evaluations of clinical responsiveness before the first cycle of chemotherapy as baseline and 1 week after the first and second cycles. Blood samples were collected to measure possible biomarkers, at the same times as CPT determinations and the day after the first cycle of chemotherapy.

\section{Assessment of CIPN}

Methods for grading clinical responsiveness to CIPN, obtained just before CPT determinations, were as follows: physician- based scale for grading classification according to NCI-CTC version 4 for sensory and motor peripheral neuropathy; numerical rating scale (NRS) of determination for pain ${ }^{5)}$ and patient neurotoxicity questionnaire(PNQ) in Japanese for various signs and symptoms ${ }^{6}$; and the subscale of neurotoxicity from functional assessment of cancer therapy/gynecologic oncology group-neurotoxicity $(\mathrm{Ntx})$ as a patient self-reporting scale for sensory and motor neuropathy ${ }^{7)}$. To compare evaluations, we converted scores to an 11-point scale from 0 to 10, with the exception of NCI-CTC. CPT examination were performed as follows: a Neurometer NS3000 (Neurotron, Baltimore, MD, USA) was used to determine the CPT values minimum current perceived in the right index finger, evoked by stimulation with a sine-wave current ${ }^{9}$. CPT range analysis was performed to compare raw CPT measurements to the normative ranges. Temperature in the examination room was stable, at approximately $25^{\circ} \mathrm{C}$.

\section{Measurements of peptide biomarkers}

Methods for measuring concentrations of peptides were as follows: plasma samples from the patient were collected and stored at $-80^{\circ} \mathrm{C}$. Plasma levels of BDNF, NGF, SP and IFN- $\gamma$ were measured with commercially available enzyme-linked immunosorbent assay (ELISA) kit for BDNF (Aviscera Bioscience, Santa Clara, CA, USA), human NGF ELISA kit (Boster Biological Technology, Pleasanton, CA, USA), SP ELISA kit (Enzo Life Sciences, Lause, Switzerland) and IFN- $\gamma$ ELISA Quantikaine Kit (R\&D Systems, Minneapolis, MN, USA), respectively. Measureable ranges were labeled to be $20-1,500$ $\mathrm{pg} / \mathrm{mL}, \quad 15^{-1}, 000 \mathrm{pg} / \mathrm{mL}, \quad 10^{-10}, 000 \mathrm{pg} / \mathrm{mL}$ and $5-1,000$ $\mathrm{pg} / \mathrm{mL}$ for BDNF, NGF, SP and IFN- $\gamma$, respectively. Intra- and inter-assay precisions for each substrate were less than $8 \%$.

\section{Ethical consideration}

This study was conducted in accordance with the Declaration of Helsinki, following approval by the institutional review boards 
A

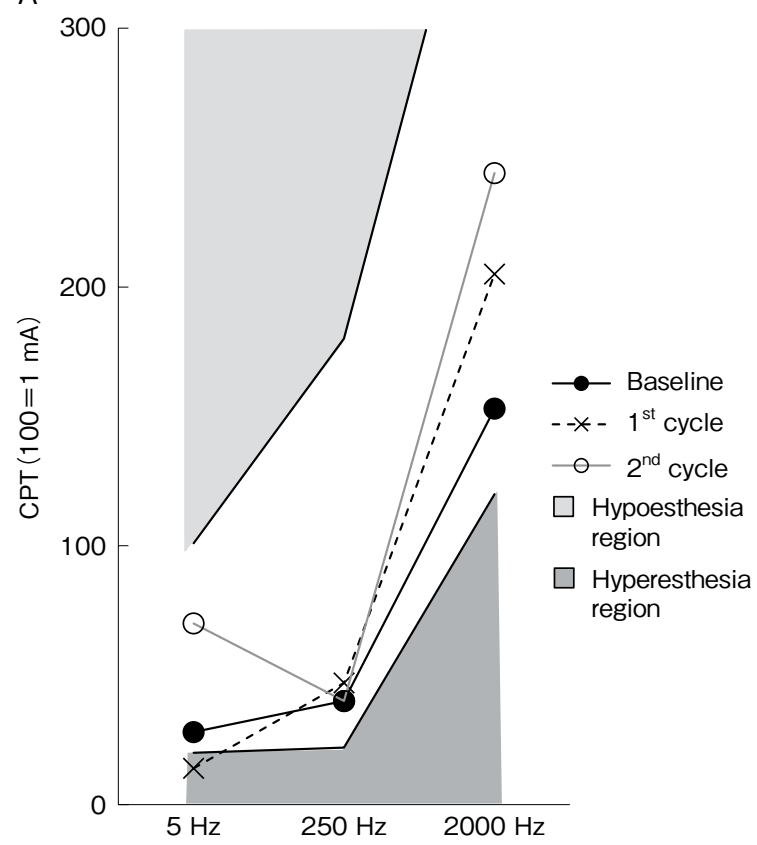

B

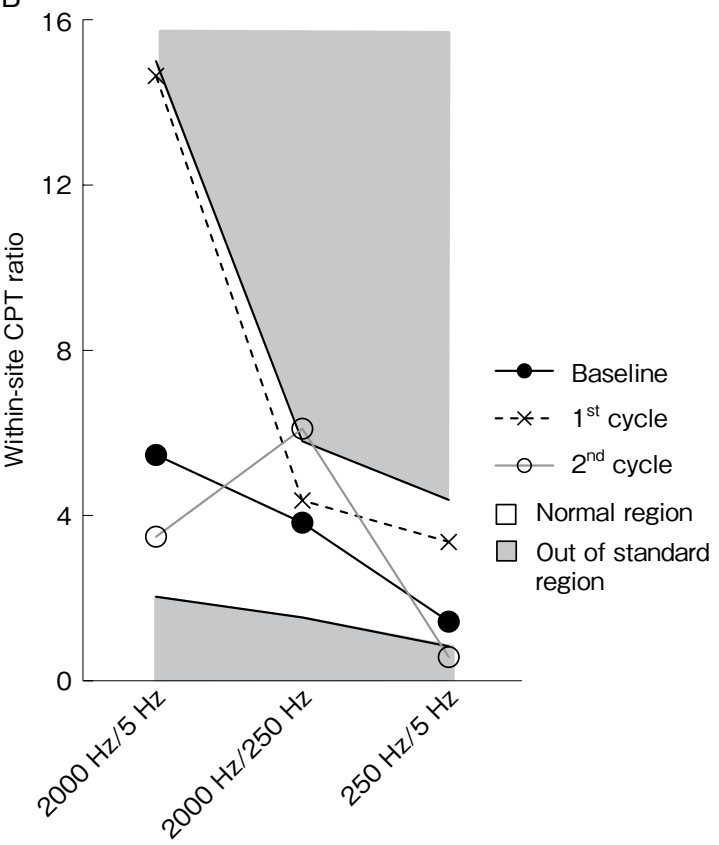

Figure 2 Raw values for current perception threshold (CPT) at $5 \mathrm{~Hz}, 250 \mathrm{~Hz}$, and 2,000 Hz (A), and within-site CPT ratios of $2,000 \mathrm{~Hz} / 5 \mathrm{~Hz}, 2,000 \mathrm{~Hz} / 250 \mathrm{~Hz}$, and $250 \mathrm{~Hz} / 5 \mathrm{~Hz}$ (B)

, baseline ; $\times$, after 1 week of the first cycle of chemotherapy ; $\bigcirc$, after 1 week of the second cycle of chemotherapy ; , hypoesthesia region and $\square$, hyperesthesia region (A) ; $\square$, normal region and $\square$, out of standard region for within-site CPT ratios (B).

of Hokkaido Pharmaceutical University School of Pharmacy and National Hospital Organization Hokkaido Cancer Center with the provision of written informed consent from the patient.

\section{Results and Discussion}

Grading scores for clinical responsiveness are shown in Figure 1. No obvious changes in physician-based scores of the NCI-CTC for sensory, patient-based PNQ and NRS were observed during the entire observation period. As for Ntx subscales, the patient complained of some numbness, tingling and discomfort in the hands. Scores in the Ntx subscale for sensory neuropathy increased after the second cycle of chemotherapy, while those for motor neuropathy decreased after the first cycle of chemotherapy.

The constant current output with the Neurometer NS3000 enabled highly reproducible measures that were unaffected by variables such as skin thickness, temperature or edema ${ }^{9)}$. As shown in Figure 2A, CPT values at $250 \mathrm{~Hz}$ were unchanged, and those at $2,000 \mathrm{~Hz}$ increased but remained within the normative range during the entire observation period. The CPT value at 5 $\mathrm{Hz}$ decreased to below the minimum normative range and qualified as hyperesthesia after 1 week of the first cycle, and then recovered into the normative range after 1 week of the second cycle of chemotherapy. The nerve differs depending on the nerve type and with the specific frequency that induces depolarization, which also depends on the diameter: the CPT at $2,000 \mathrm{~Hz}$ corresponds to sensory impairment of large myelinated fibers $(\mathrm{A} \beta$ fibers $)$, the $\mathrm{CPT}$ at $250 \mathrm{~Hz}$ to that of small myelinated fibers (A $\delta$ fibers), and the $\mathrm{CPT}$ at $5 \mathrm{~Hz}$ to that of an unmyelinated nerve $(\mathrm{C} \text { fiber })^{9)}$. Meanwhile, within-site CPT ratio analysis offers a sensitive analytical method for measuring the ratio within the measurement range ${ }^{9)}$. As shown in Figure 2B, within-site CPT ratios at baseline for $2,000 \mathrm{~Hz} / 5 \mathrm{~Hz}, 2,000$ $\mathrm{Hz} / 250 \mathrm{~Hz}$, and $250 \mathrm{~Hz} / 5 \mathrm{~Hz}$ and those ratios after 1 week of the first cycle of chemotherapy were within the normal ranges. However, those ratios after 1 week of second cycles of chemotherapy at $2,000 \mathrm{~Hz} / 250 \mathrm{~Hz}$ and $250 \mathrm{~Hz} / 5 \mathrm{~Hz}$ shifted into abnormal ranges. Considering these results from CPT examinations, the patient was thought to have initial paresthesia as sensory CIPN, especially in the hands, with a slight increase in grading scores of the Ntx subscale after 1 week of the second cycle of chemotherapy (Figure 1). This paresthesia was expected to correspond to the observed initial changes in abnormalities of within-site CPT ratios at $2,000 \mathrm{~Hz} / 250 \mathrm{~Hz}$ and $250 \mathrm{~Hz} / 5 \mathrm{~Hz}$. Our observations of sudden changes to balances in within-site CPT ratios were thought to induce the unpleasant sensation. These results indicate slight impairment in $\mathrm{A} \beta$ and $\mathrm{C}$ fibers, even if each raw value for CPT was observed to be almost within the normal range at this time (Figure 2A). Our observed changes in raw values at $5 \mathrm{~Hz}$ indicated that temporary sensitization of $\mathrm{C}$ fibers may have resulted from slight damage or inflammation after the first cycle of therapy, followed by rapid recovery to normal. These changes in $\mathrm{C}$ fibers were thought to correlate with the flash increases in SP levels, since $\mathrm{C}$ fibers contain high proportion of $\mathrm{SP}^{11)}$.

As shown in Figure 3, reduced circulating levels of $\operatorname{NGF}(A)$ and increased levels of SP (B) were observed on the next day of the second cycle of chemotherapy. Increased levels of $\operatorname{BDNF}(\mathrm{C})$ and IFN- $\gamma$ (D) were observed after 1 week of the second cycle of chemotherapy. Considering these results, the observed changes 

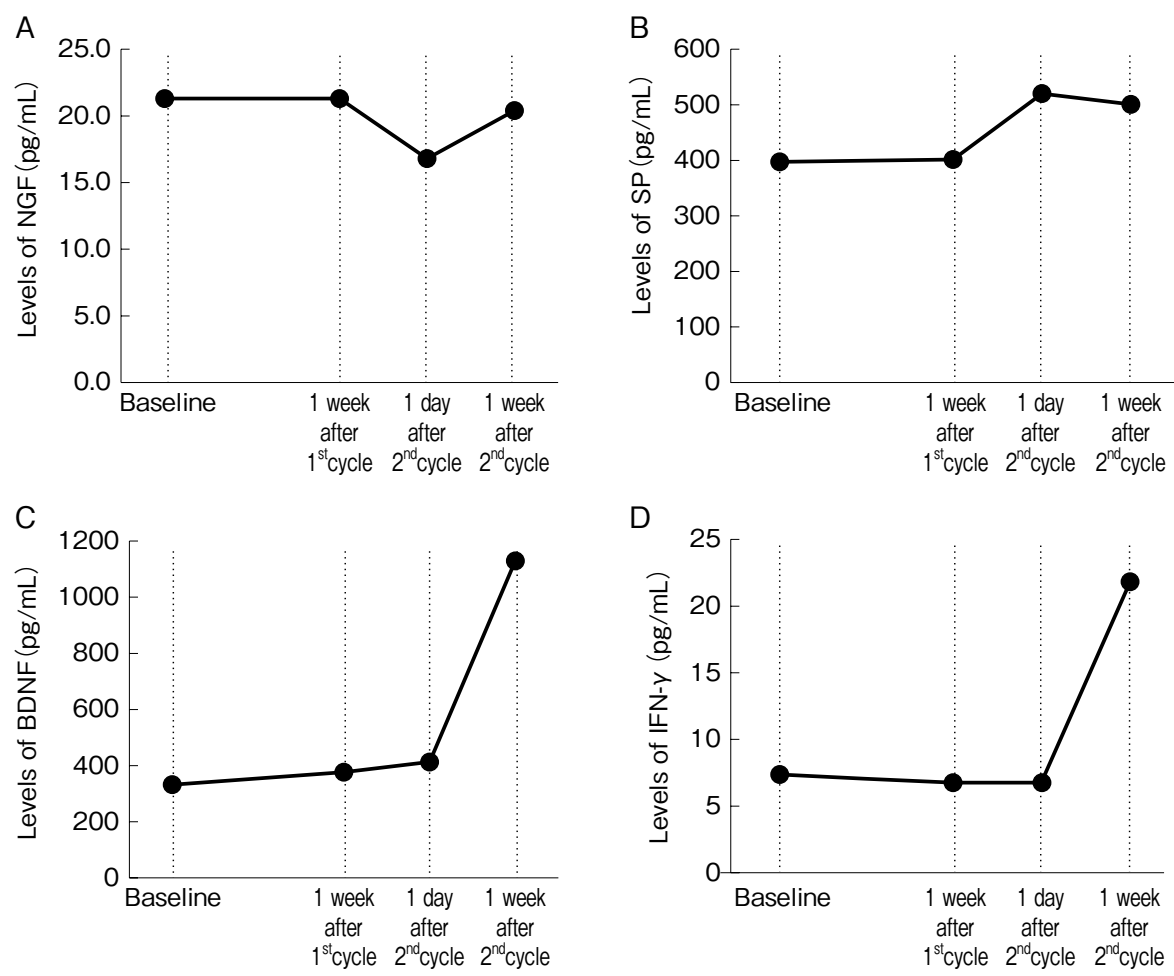

Figure 3 Change in circulating blood levels of biomarkers

Levels of nerve growth factor (A), substance $\mathrm{P}$ (B), brain-derived neurotropic factor (C) and interferon- $\gamma$ (D). Measurements were observed at before and the next day of the first cycle of chemotherapy and 1 week after the first and second cycles of chemotherapy. NGF, nerve growth factor ; SP, substance P ; BDNF, brain-derived neurotropic factor ; IFN- $\gamma$, interferon- $\gamma$.

in CPT values were accompanied by increases in BDNF and IFN- $\gamma$ levels and were preceded by rapid changes in SP and also in NGF levels. Decreased NGF levels reportedly correlate with the severity of CIPN caused by platinum-derived drugs ${ }^{14}$ and are thought to be early predictors of CIPN ${ }^{15)}$. The grade of CIPN severity for our patient was not considered serious during observation. This was because observed NGF levels decreased slightly on the day after the second cycle of therapy, similar to previously reported values for oxaliplatin-induced $\operatorname{CIPN}^{14)}$, but decreased transiently and quickly recovered by 1 week after the second cycle of therapy (Figure 3A). These rapid changes may correspond to NGF and mostly correspond to $\mathrm{A} \delta$ fibers and $\mathrm{C}$ fibers $^{10)}$. However, such early activation and impairment may have occurred in $\mathrm{C}$ fibers rather than $\mathrm{A} \delta$ fibers in the patient, because the observation of unchanged CPT values at $250 \mathrm{~Hz}$ indicates the absence of sensory impairment of $\mathrm{A} \delta$ fibers (Figure 2A).

Risks for side effects such as hyperalgesia may correlate with levels of NGF, SP and BDNF. Endogenous BDNF in the dorsal horn contributes to hyperalgesia, is associated with peripheral inflammation in the early stage, and is accompanied by NGFinduced up-regulation of $\mathrm{SP}^{12}$. Exogenous BDNF indirectly suppresses sensory neuronal transmission through activation of $\gamma$-aminobutyric acid (GABA) type B receptors located on the terminals of sensory neurons in normal conditions ${ }^{10)}$. Increased BDNF is expected to promote and regulate excess activation. Thus, recovery of sensitized $\mathrm{C}$ fibers after the second cycle of therapy may be influenced by the observed sudden increase in BDNF levels (Figure 3C).

In patients with allodynia complicating CIPN, distinguishing each grade in the NCI-CTC is difficult. However, in allodynia that underlies sustained neuropathic pain, BDNF shifts the neuronal anion gradient ${ }^{13)}$ and switches the normal neuronal suppressive effects caused by GABA to the opposite effect including hyperexcitability of neurons ${ }^{16)}$. IFN- $\gamma$ up-regulates purinoceptors such as $\mathrm{P} 2 \mathrm{X} 4 \mathrm{R}$ in microglia, which reportedly induce P2X4R-dependent allodynia through a release of BDNF from microglia ${ }^{17)}$. This patient showed no indication of allodynia during the observation period, considering the absences of clinical responsiveness and sustained decreases in thresholds for individual CPT values. If increased BDNF is sustainably observed and accompanied by chronic increases in IFN- $\gamma$ levels, the risk of IFN- $\gamma$-related allodynia would increase in the patient.

Based on these results, individual levels of NGF, SP and BDNF have the potential to be monitored as possible candidate biomarkers for sensory impairment in early-stage CIPN. This was a pilot case to show the importance of these evaluations in combination, which may compensate for difficulties in assessing clinical responsiveness of CIPN. These simple quantitative evaluations are likely to prove beneficial in deciding changes to the next regimen to prevent exacerbation of neuropathy and to develop available treatment for CIPN. Accumulation of these data is necessary to provide safe and effective chemotherapy. 


\section{Acknowledgement}

This research was supported by funds from an Education and Research Grant from the Hokkaido Pharmaceutical University and a Research Grant from Sawai Pharmaceutical Company Limited.

\section{Conflict of Interest}

The authors have no conflicts of interest to disclose in relation to this article.

\section{References}

1) Goldberg RM, Sargent DJ, Morton RF, Fuchs CS, Ramanathan RK, Williamson SK, et al. A randomized controlled trial of fluorouracil plus leucovorin, irinotecan, and oxaliplatin combinations in patients with previously untreated metastatic colorectal cancer. J Clin Oncol. 2004; 22(1) : 23-30.

2) Postma TJ, Heimans JJ, Muller MJ, Ossenkoppele GJ, Vermorken JB, Aaronson NK. Pitfalls in grading severity of chemotherapy-induced peripheral neuropathy. Ann Oncol. 1998; 9(7) : 739-44.

3) Tournigand C, Cervantes A, Figer A, Lledo G, Flesch M, Buyse M, et al. OPTIMOX1 : a randomized study of FOLFOX4 or FOLFOX7 with oxaliplatin in a stop-and-Go fashion in advanced colorectal cancer--a GERCOR study. J Clin Oncol. 2006; 24(3) : 394-400.

4) Simozuma K, Ohashi Y, Takeuchi A, Aranishi T, Morita S, Kuroi K, et al. Feasibility and validity of the Patient Neurotoxicity Questionnaire during taxane chemotherapy in a phase $\mathrm{III}$ randomized trial in patients with breast cancer: N-SAS BC 02. Support Care Cancer. 2009; 17 (12) : 1483-91. doi : 10.1007/s00520-009-0613-7.

5) Jensen MP. The validity and reliability of pain measures in adults with cancer. J Pain. 2003; 4 (1) : 2-21.

6) Hausheer FH, Schilsky RL, Bain S, Berghorn EJ, Lieberman F. Diagnosis, management, and evaluation of chemotherapy-induced peripheral neuropathy. Semin Oncol. 2006; 33(1) : 15-49.

7) Fumimoto $\mathrm{H}$, Kobayashi $\mathrm{K}$, Chang $\mathrm{CH}$, Eremenco S, Fujiki $\mathrm{Y}$, Uemura $\mathrm{S}$, et al. Cross-cultural validation of an international questionnaire, the
General Measure of the Functional Assessment of Cancer Therapy scale (FACT-G), for Japanese. Qual Life Res. 2001; 10 (8) : 701-9.

8) Takekuma K, Ando F, Niino N, Shimokata H. Prevalence of hyperesthesia detected by current perception threshold test in subjects with glucose metabolic impairments in a community. Intern Med. $2002 ; 41(12)$ : 1124-9.

9) Katims JJ, Long DM, Ng LK. Transcutaneous nerve stimulation: frequency and waveform specificity in humans. Appl Neurophysiol. 1986; 49 (1-2) : 86-91.

10) Khan N, Smith MT. Neurotrophins and neuropathic pain: role in pathobiology. Molecules. 2015; 20 (6) : 10657-88. doi : 10.3390/ molecules200610657.

11) Millan MJ. The induction of pain: an integrative review. Prog Neurobiol. 1999; 57 (1) : 1-164.

12) Skoff AM, Adler JE. Nerve growth factor regulates substance $P$ in adult sensory neurons through both TrkA and p75 receptors. Exp Neurol. $2006 ; 197$ (2) : 430-6.

13) Tsuda M, Inoue K. Neuron-microglia interaction by purinergic signaling in neuropathic pain following neurodegeneration. Neuropharmacology. 2015 Aug 29. doi: 10.1016/j. neuropharm. 2015.08.042. [Epub ahead of print]

14) De Santis S, Pace A, Bove L, Cognetti F, Properzi F, Fiore M, et al. Patients treated with antitumor drugs displaying neurological deficits are characterized by a low circulating level of nerve growth factor. Clin Cancer Res. 2000; 6(1) : 90-5.

15) Cavaletti G, Bogliun G, Marzorati L, Zincone A, Piatti M, Colombo N, et al. Early predictors of peripheral neurotoxicity in cisplatin and paclitaxel combination chemotherapy. Ann Oncol. 2004; 15 (9): 1439-42.

16) Coull JA, Beggs S, Boudreau D, Boivin D, Tsuda M, Inoue K, et al. BDNF from microglia causes the shift in neuronal anion gradient underlying neuropathic pain. Nature. 2005; 438(7070) : 1017-21.

17) Tsuda M, Shigemoto-Mogami Y, Koizumi S, Mizokoshi A, Kohsaka S, Salter MW, et al. P2X4 receptors induced in spinal microglia gate tactile allodynia after nerve injury. Nature. 2003; 424 (6950) : 778-83. 